\title{
Carbon-nanotube-based quantum pump in the presence of a superconducting lead
}

\author{
Yadong Wei \\ Department of Physics, School of Science, Shenzhen University, Shenzhen 518060, China \\ Jian Wang* \\ Department of Physics, The University of Hong Kong, Pokfulam Road, Hong Kong, China
}

(Received 13 June 2002; revised manuscript received 3 September 2002; published 27 November 2002)

\begin{abstract}
Parametric electron pump through superconductor-carbon-nanotube based molecular devices was investigated. It is found that a dc current, which is assisted by resonant Andreev reflection, can be pumped out from such molecular device by a cyclic variation of two gate voltages near the nanotube. The pumped current can be either positive or negative under different system parameters. Due to the Andreev reflection, the pumped current has the double peak structure around the resonant point. The ratio of pumped current of N-SWNT-S system to that of N-SWNT-N system $\left(I^{\mathrm{NS}} / I^{\mathrm{N}}\right)$ is found to approach four in the weak pumping regime near the resonance when there is exactly one resonant level at Fermi energy inside the energy gap. Numerical results confirm that in the weak pumping regime the pumped current is proportional to the square of the pumping amplitude $V_{p}$, but in the strong pumping regime the pumped current has the linear relation with $V_{p}$. For the pumped current via two resonant levels, we found that the current reversal behavior, i.e., the direction of the current reverses when the Fermi energy is varied. This is different from the one dimensional double barrier structure. Our numerical results also predict that pumped current can be obtained more easily by using zigzag tube than by using armchair tube. We have also derived the formula for the total current in the presence of both pumping potential and external bias and applied our formula to the armchair and zigzag carbon nanotubes.
\end{abstract}

DOI: 10.1103/PhysRevB.66.195419 PACS number(s): 73.63.Fg, 85.35.Kt, 73.40.Gk, 74.50.+r

\section{INTRODUCTION}

Physics of parametric electron pump has attracted great attention. Classical pumps had been fabricated about a decade ago. ${ }^{1,2}$ Recently, quantum-dot-based quantum pump has been the subject of both experimental ${ }^{3}$ and theoretical ${ }^{4-19,22}$ investigations. The quantum pump generates current due to the cyclic variation of at least two system parameters while maintaining zero bias. As the charge pumped out of the system, it also produces the Joule heat along with the dissipation. Recently, Avron et al. ${ }^{23}$ have derived the lower bound for the dissipation. This naturally leads to the concept of optimal pump. To search for an optimal pump, the heat current and the shot noise of quantum pump have been investigated using the time-dependent scattering matrix theory. ${ }^{24-26}$ Very recently, to explain the experimentally observed anomaly, ${ }^{3}$ the finite frequency pumping theory has been developed. ${ }^{27,28}$ The adiabatic pumping theory has also been extended to account for the Andreev reflection in the presence of superconducting lead, ${ }^{18,29}$ strong electron interaction in the Kondo regime, ${ }^{30}$ and spin polarized pumped current when the ferromagnetic leads are present. ${ }^{31}$ Due to the peculiar electronic properties of carbon nanotube (CNT), ${ }^{32-40}$ CNT-based parametric electron pump has been investigated as a prototypical nanometer-scale molecular device. ${ }^{14}$ It would be interesting to further explore how does Andreev reflection modify the quantum interference of CNT based quantum pump in the presence of superconducting lead. It is well known that in the presence of normal conductorsuperconductor (NS) interface, an incoming electronlike excitation can be Andreev reflected as a holelike excitation. ${ }^{41}$ In this paper, we will study a hybrid structure where both carbon nanotube and superconducting lead are present and examine the interplay between the electronic properties of CNT and superconductivity. Specifically, we investigate a parametric quantum pump that consists of a finite sized single wall carbon nanotube (SWNT) connected to one normal left lead $(\mathrm{N})$ and one superconducting right lead (S), i.e., the parametric quantum pump of N-SWNT-S system. Two pumping driving forces $X_{1}(t)$ and $X_{2}(t)$ are established by applying cyclic, time-dependent voltages to two metallic gates, which are capacitively coupled to the SWNT (see inset of Fig. 1). We have used nonequilibrium Green's function approach $^{42-44}$ to calculate the pumped current. We found that in the presence of superconducting lead the pumped current is four times as that of the corresponding normal system in

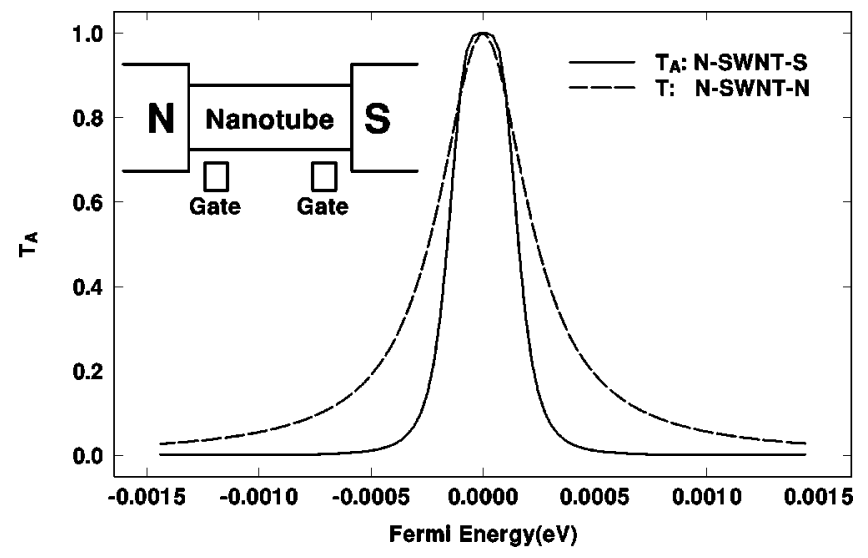

FIG. 1. Andreev reflection coefficient $T_{A}$ as a function of Fermi energy for N-SWNT-S system (solid line) and transmission coefficient $T$ as a function of Fermi energy for N-SWNT-N device (long dashed line). The SWNT is an armchair $(5,5)$ metallic tube. Inset: a schematic plot of the molecular device. 
the weak pumping regime. As the pumping amplitude increases, the dependence of pumped current crosses over from quadratic to the linear dependence. Due to the Andreev reflection, the pumped current exhibits double peak structure for single resonant level in line with the chemical potential $\mu_{s}$ of the superconducting lead. When $\mu_{s}$ is in the middle of two resonant levels, another type of Andreev reflection occurs where an electron coming from normal lead tunnels via the lower resonant level and Andreev reflected as a hole through the upper resonant level with a Cooper pair created in the superconducting lead. For this two level Andreev reflection, the pumped current shows remarkable parity effect that the direction of pumped current near one resonant level is opposite to that of the other level. This is different from the one dimensional double barrier structure. Our numerical results show that it is much easier to pump current through a zigzag structure than an armchair structure. Parametric pump generates current at zero external bias. It would be interesting to see the interplay of the role played by the pumping potential and the external bias if the external bias is applied. ${ }^{19}$ In this paper, we have also derived a formula for the current driven by both the pumping potential and external bias to the NS system which recovers the formula obtained by Entin-Wohlman et al. ${ }^{19}$ when the lead becomes normal. Numerical results are also provided and discussed.

\section{THEORY}

In this work, we assume that the variation of the pumping potentials are very slow and the adiabatic approximation is appropriate. We further assume that there is an external bias $V_{L}$ applied on the left normal lead. ${ }^{19}$ As a result, the total current contains two parts, the average current $I_{v}$ due to the external bias voltage and the pumped current $I_{p}$ caused by the pumping signal,

$$
I^{\mathrm{NS}}=I_{v}+I_{p},
$$

where

$$
I_{v}=\frac{1}{\tau} \int_{0}^{\tau} i_{A}(t) d t
$$

and the instantaneous current is ${ }^{20,21}$

$$
\begin{aligned}
i_{A}(t)= & -2 q \int \frac{d E}{2 \pi} \operatorname{Tr}\left[\Gamma_{L} G_{12}^{r} \Gamma_{L} G_{21}^{a}\right]\left[f\left(E-q V_{L}\right)\right. \\
& \left.-f\left(E+q V_{L}\right)\right],
\end{aligned}
$$

where $G_{12}^{r}$ is defined below. Now we derive the pumped current. Using the distribution function, the total charge in the scattering region during the pumping is given by

$$
Q(x, t)=-i \int(d E / 2 \pi)\left[\mathbf{q} \mathbf{G}^{<}(E,\{X(t)\})\right]_{x x},
$$

where $\mathbf{q}=q \sigma_{z}$ with $\sigma_{z}$ the Pauli matrix and $\mathbf{G}^{<}$is the lesser Green's function in real space, $x$ labels the position, and $\{X(t)\}$ describes a set of external parameters which facili- tates the pumping process. $\mathbf{G}^{<}$is related to the retarded and advanced Green's functions $\mathbf{G}^{r}$ and $\mathbf{G}^{a}$,

$$
\mathbf{G}^{<}(E,\{X\})=\mathbf{G}^{r}(E,\{X\}) \mathbf{\Sigma}^{<}(E) \mathbf{G}^{a}(E,\{X\}) .
$$

In the low frequency limit, the retarded Green's function in real space is given by

$$
\mathbf{G}^{r}(E,\{X\})=\frac{1}{E-\mathbf{H}_{d}-\mathbf{X}_{p p}-\mathbf{\Sigma}^{r}},
$$

where $\boldsymbol{\Sigma}^{r}$ (see below) is the self energy. In the above equations, $\mathbf{G}^{r, a,<}$ denotes a $2 N \times 2 N$ matrix with matrix elements $G_{\gamma \gamma^{\prime}, i j}^{r, a<}$ with $\gamma$ denoting the spin degrees of freedom and $i$ for the orbital degrees of freedom. Here $\gamma=1,2$ labels electron and hole (electron below the Fermi surface with opposite momentum) and $N$ is the number of grid points in real space in the tight binding representation. $\mathbf{X}_{p p}=V_{p p} \sigma_{z}$. In real space representation, $V_{p p}$ is a diagonal matrix describing the variation of the potential landscape due to the external pumping parameter $X$. In order for a parametric electron pump to function at low frequency, we need simultaneous variation of two or more system parameters controlled by gate voltages: $X_{i}(t)=V_{i 0}+V_{i p} \sin \left(\omega t+\phi_{i}\right)$. Hence, in our case, the potential due to the gates can be written as $\mathbf{X}_{p p}=\Sigma_{i} V_{i} \boldsymbol{\Delta}_{i}$, where $\boldsymbol{\Delta}_{i}=\Delta_{i} \sigma_{z}$ is the potential profile due to each pumping potential. If the time variation of these parameters are slow, i.e., for $V(t)=V_{0}+\delta V \sin (\omega t)$, then the charge (including that of both electron and hole) of the system coming from the left lead due to the infinitesimal change of the system parameter $(\delta X \rightarrow 0)$ is

$$
d Q_{p}(t)=\sum_{i} \partial_{X_{i}} \operatorname{Tr}[Q(x, t)] \delta X_{i}(t),
$$

it is easily seen that the total charge in the system in a period is zero which is required for the charge conservation. To calculate the pumped current, we have to find the electron $d Q_{p, e}$ passing through left lead due to the change of the system parameters. Using the Dyson equation $\partial_{X_{i}} \mathbf{G}^{r}$ $=\mathbf{G}^{r} \Delta_{i} \mathbf{G}^{r}$, the above equation becomes

$$
\begin{aligned}
d Q_{p}(t)= & \sum_{j} \int \frac{d E}{2 \pi} \operatorname{Tr}\left[\mathbf{q} \mathbf{G}^{r} \boldsymbol{\Delta}_{j} \mathbf{G}^{r} \boldsymbol{\Sigma}^{<} \mathbf{G}^{a}\right. \\
& \left.+\mathbf{q} \mathbf{G}^{r} \boldsymbol{\Sigma}^{<} \mathbf{G}^{a} \boldsymbol{\Delta}_{j} \mathbf{G}^{a}\right] \delta X_{j}(t) \\
= & -\int \frac{d E}{2 \pi} \sum_{j} \operatorname{Tr}\left[\mathbf{q} \mathbf{G}^{a} \partial_{E} \boldsymbol{\Sigma}^{<} \mathbf{G}^{r} \boldsymbol{\Delta}_{j}\right] \delta X_{j}(t),
\end{aligned}
$$

where we have integrated by part in the last step. Hence the pumped electron during the time interval is

$$
d Q_{p, e}(t)=-q \int \frac{d E}{2 \pi} \sum_{j} \operatorname{Tr}\left[\mathbf{G}^{a} \partial_{E} \boldsymbol{\Sigma}^{<} \mathbf{G}^{r} \boldsymbol{\Delta}_{j}\right]_{11} \delta X_{j}(t) .
$$

Here the $\operatorname{Tr}$ is over the orbital degrees of freedom. Using the fact that ${ }^{20}$ 


$$
\mathbf{\Sigma}^{<}=\left(\begin{array}{cc}
i \Gamma_{L} f\left(E-q V_{L}\right) & 0 \\
0 & i \Gamma_{L} f\left(E+q V_{L}\right)
\end{array}\right)
$$

and the definition of $\boldsymbol{\Delta}_{i}$, we finally obtain

$$
\begin{aligned}
I_{p}= & \frac{q}{\tau} \int_{0}^{\tau} d t \sum_{j} \int \frac{d E}{2 \pi}\left\{\partial_{E} f(E-q V) \operatorname{Tr}\left[G_{11}^{a} \Gamma_{L} G_{11}^{r} \Delta_{j}\right]\right. \\
& \left.-\partial_{E} f\left(E+q V_{L}\right) \operatorname{Tr}\left[G_{12}^{a} \Gamma_{L} G_{21}^{r} \Delta_{j}\right]\right\} \frac{d X_{j}}{d t} .
\end{aligned}
$$

Equation (1), with Eqs. (2), (3), and (11) forms the superconducting analog of Ref. 19. If the external bias is zero, we obtain $^{4,45}$

$$
I^{\mathrm{NS}}=\frac{q \omega}{2 \pi} \int_{0}^{\tau} d t\left[\frac{d N_{L}}{d X_{1}} \frac{d X_{1}}{d t}+\frac{d N_{L}}{d X_{2}} \frac{d X_{2}}{d t}\right],
$$

where the quantity $d N_{L} / d X_{j}$ is the partial density of states (DOS), called the injectivity, ${ }^{46,47}$ of the left lead,

$$
\frac{d N_{L}}{d X_{j}}=\frac{d N_{L}^{e}}{d X_{j}}-\frac{d N_{L}^{h}}{d X_{j}},
$$

with

$$
\frac{d N_{L}^{e}}{d X_{j}}=-\int \frac{d E}{2 \pi}\left(-\partial_{E} f(E)\right) \operatorname{Tr}\left[G_{11}^{a} \Gamma_{L} G_{11}^{r} \Delta_{j}\right]
$$

and

$$
\frac{d N_{L}^{h}}{d X_{j}}=-\int \frac{d E}{2 \pi}\left(-\partial_{E} f(E)\right) \operatorname{Tr}\left[G_{12}^{a} \Gamma_{L} G_{21}^{r} \Delta_{j}\right],
$$

where $j=1$ or $2 . d N_{L}^{e} / d X_{j}$ describes the number of electrons coming from the left lead and exiting the system as electrons due to the external parameter $X_{j} . d N_{L}^{h} / d X_{j}$ describes the number of holes coming from the left lead and exiting the system as electrons due to the external parameter $X_{j} \cdot{ }^{47} G_{11}$ and $G_{12}$ are the matrix elements of the $2 \times 2 \mathrm{Nambu}^{48}$ representation and can be expressed as ${ }^{20}$ :

$$
G_{11}^{r}(E)=\left[E-H_{d}-V_{p p}-\mathbf{\Sigma}_{11}^{r}-\Sigma_{12}^{r} A^{r} \mathbf{\Sigma}_{21}^{r}\right]^{-1}
$$

and

$$
A^{r}=\left[E+H_{d}^{*}+V_{p p}-\boldsymbol{\Sigma}_{22}^{r}\right]^{-1} .
$$

Once the electron and hole Green's function $G_{11}^{r}$ and $A^{r}$ were obtained, $G_{12}^{r}$ is calculated by

$$
G_{12}^{r}=G_{11}^{r} \boldsymbol{\Sigma}_{12}^{r} A^{r} .
$$

Here $\Gamma_{L, R}=-2 \operatorname{Im}\left[\Sigma_{L, R}^{r}\right]$ is the linewidth function and $\boldsymbol{\Sigma}^{r}$ $=\boldsymbol{\Sigma}_{L}^{r}+\boldsymbol{\Sigma}_{R}^{r}$ is the total self-energy given by

$$
\mathbf{\Sigma}_{L}^{r}=\left(\begin{array}{ll}
\Sigma_{L}^{r} & 0 \\
0 & -\Sigma_{L}^{a}
\end{array}\right)
$$

where $\Sigma_{\alpha}^{r} \equiv P_{\alpha}-i \Gamma_{\alpha} / 2$ is the self-energy of the lead $\alpha$ in the normal case. Here $P_{\alpha}$ is the real part and $\Gamma_{\alpha}$ is the linewidth function. The self-energy for the superconducting lead is

$$
\boldsymbol{\Sigma}_{R}^{r}=\left(\begin{array}{ll}
P_{R}-i \Gamma_{R} \beta_{1} / 2 & i \Gamma_{R} \beta_{2} / 2 \\
i \Gamma_{R} \beta_{2} / 2 & -P_{R}-i \Gamma_{R} \beta_{1} / 2
\end{array}\right),
$$

where $\beta_{1}=\nu E / \sqrt{E^{2}-\Delta^{2}}, \beta_{2}=\nu \Delta / \sqrt{E^{2}-\Delta^{2}}$ with $\nu=1$ for $E>-\Delta$ and $\nu=-1$ for $E<-\Delta$. Here $\Delta$ is the gap energy of superconducting lead and chemical potential of superconducting lead $\mu_{s}$ has been set to zero. In the above equations, $H_{d}$ is the Hamiltonian of CNT. It is a $N \times N$ matrix, where $N$ is the total number of carbon atoms. $V_{p p}$ is a diagonal matrix describing the variation of the CNT potential landscape due to the external pumping potentials $X_{1}(t)$ and $X_{2}(t)$. In this work, we choose the two pumping potentials to be $X_{1}(t)$ $=V_{10}+V_{1 p} \sin (\omega t)$ and $X_{2}(t)=V_{20}+V_{2 p} \sin (\omega t+\phi)$, where $\phi$ is phase difference, $\omega$ is the pumping frequency and $V_{1 p}$ and $V_{2 p}$ are the pumping amplitudes. To simplify the numerical calculation, we mimic the gate effects by simply adding the potential $V_{p p}=V_{1} \Delta_{1}+V_{2} \Delta_{2}$ to the SWNT where $\Delta_{i}$ is the potential profile function and can be set to be unit for the gate region, zero otherwise. A more accurate study requires a numerical solution of the Poisson equation with the gates providing the appropriate boundary conditions.

\section{RESULT AND DISCUSSION}

We now apply our theory to calculate the current for the N-SWNT-S parametric pump. For simplicity, the SWNT is modeled with the nearest neighbor $\pi$-orbital tight-binding model with bond potential $V_{p p \pi}=-2.75 \mathrm{eV}$. This model gives a reasonable, qualitative description of the electronic and transport properties of carbon nanotubes. ${ }^{49,50}$ Recently, a S-SWNT-S device has been studied experimentally. ${ }^{36}$ By tuning the transparency of the device, clear signals of Andreev reflection were observed. The dependence of the Andreev current on the device transparency, the behavior of the differential resistance in the subgap region, as well as the observed low-temperature resistance anomaly ${ }^{36}$ can all be explained theoretically using the $\pi$-orbital tight-binding model. ${ }^{51}$ We assume that the SWNT is weakly coupled to the electrodes so that the pumping process is mediated by the resonant tunneling. We further assume that strong electronelectron interactions may be neglected. Without losing generality, we set the energy gap of the superconducting lead to be $1.45 \mathrm{meV}$ (the gap of Niobium). We also apply the wide bandwidth limit for the self-energy ${ }^{42}$ and consider the symmetric pumping, i.e., $V_{10}=V_{20}=V_{0}$ and $V_{1 p}=V_{2 p}=V_{p}$. In the absence of pumping we have $V_{p}=0$ which forms a symmetric double barrier with barrier height $V_{0}$ in the finite size nanotube. As a result, the discrete resonant levels are established within the double barrier structure. By adjusting $V_{0}$, we can control the positions of energy levels inside the energy gap $\Delta$. Since the pumped current is proportional to $\omega$, we set $\omega=1$ for convenience. We also set $\hbar=2 m=-q$ $=1$. When pumping frequency $\omega=100 \mathrm{MHz}$, which is close to the frequency used in Ref. 3, the unit for the pumped current is $1.6 \times 10^{-11}$ A. Finally, we do not consider the finite temperature effect and hence set temperature to zero.

We now consider the case when external bias is absent and compare our result $I^{\mathrm{NS}}$ with that of normal case. First, we consider an armchair $(5,5)$ SWNT with 93 layers of car- 


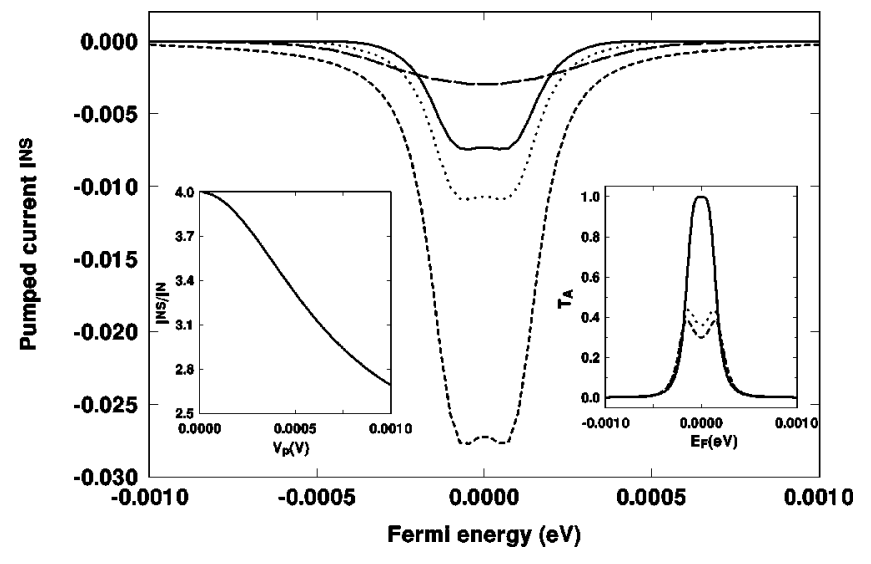

FIG. 2. The pumped current $I^{\mathrm{NS}}$ versus Fermi energy for N-SWNT-S device at different pumping amplitudes: $V_{p}$ $=0.0014 \mathrm{~V}$ (solid line), $V_{p}=0.002 \mathrm{~V}$ (dotted line), and $V_{p}$ $=0.005 \mathrm{~V}$ (dashed line). The long dashed line is the pumped current $I^{\mathrm{N}}$ versus Fermi energy for the corresponding N-SWNT-N device (when $\Delta=0$ ) at $V_{p}=0.0014 \mathrm{~V}$. The left inset, $I^{\mathrm{NS}} / I^{\mathrm{N}}$ versus $V_{p}$ at resonant point. The right inset, Andreev reflection coefficient $T_{A}$ versus $E_{F}$ with $V_{p}=0.005 \mathrm{~V}$ at different pumping moments, $t$ $=3 \pi / 4$ (solid line), $t=0.95 \times 3 \pi / 4$ (dotted line), and $t=1.05$ $\times 3 \pi / 4$ (dashed line).

bon atoms (total 930 atoms). The two metallic gates that provide the pumping driving forces are located near the two ends of the SWNT from 10th to 28th layer, and 66th to 84th layer. We have chosen $V_{0} \approx 2.75 \mathrm{~V}$ so that there is only one resonant level in the energy gap and the level is aligned with the chemical potential of the superconducting lead in the absence of pumping voltage $V_{p}=0$. Figure 1 shows the transmission coefficient versus Fermi energy $E_{F}$. For comparison, the transmission coefficient for corresponding normal system (when $\Delta=0$ ) is also plotted. We see that for normal system, the transmission coefficient (dashed line) has Lorentzian line shape. In the presence of superconducting lead, the transmission peak (solid line) is flattened and narrowed. Figure 2 depicts the pumped current $I^{\mathrm{NS}}$ versus the Fermi energy for different pumping amplitudes $V_{p}$ with $\phi$ $=\pi / 2$ and $\Gamma_{L}=\Gamma_{R}=0.0136 \mathrm{eV}$. Several interesting observations are in order. (1) The pumped current has large amplitude only near the resonant level showing clearly a resonance assisted behavior. (2) The amplitude of pumped current has double-peak structure around the resonant level. We notice that the double-peak for armchair structure is fairly symmetric which is different from one dimensional double barrier structure in Ref. 18 where an asymmetric double-peak is observed. To understand this behavior, we plot the Andreev reflection coefficient versus $E_{F}$ at different times $t$ during the pumping cycle in the right inset of Fig. 2. The Andreev reflection coefficient gives large value only around the two pumping instants: $t=3 \pi / 4$ and $t=7 \pi / 4$ because at such moments the energy level of the SWNT is just in line with the chemical potential of the superconducting lead so that an excitation of hole can be reflected when there is an incident election near the Fermi surface, and vice verse. At other moments, Andreev reflection coefficient is very small and contributes little to the current integral in the time cycle. In the

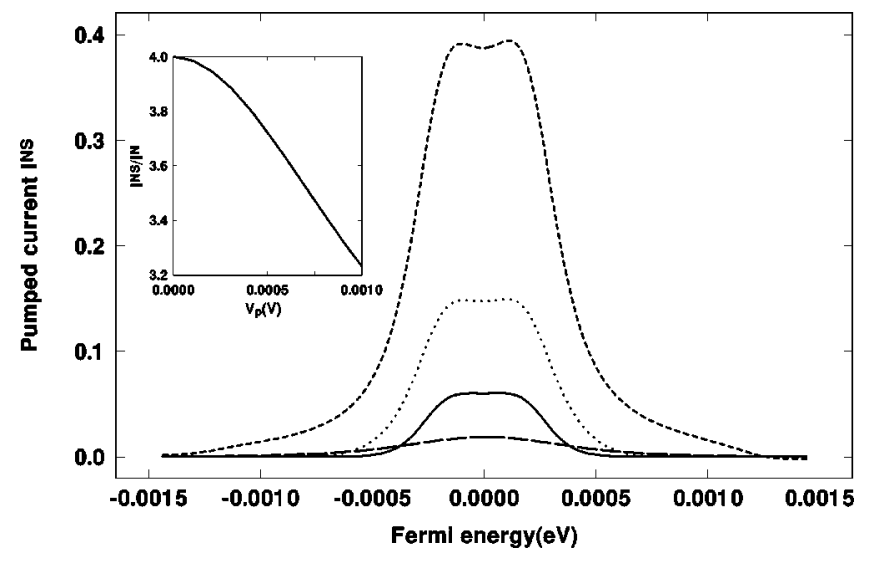

FIG. 3. The pumped current $I^{\mathrm{NS}}$ versus Fermi energy for N-SWNT-S device at different pumping amplitudes: $V_{p}=0.001 \mathrm{~V}$ (solid line), $V_{p}=0.002 \mathrm{~V}$ (dotted line) and $V_{p}=0.005 \mathrm{~V}$ (dashed line). The long dashed line is for the pumped current $I^{\mathrm{N}}$ versus Fermi energy of the corresponding N-SWNT-N device at $V_{p}$ $=0.001 \mathrm{~V}$. Here the SWNT is a zigzag $(10,0)$ nanotube. The left inset, $I^{\mathrm{NS}} / I^{\mathrm{N}}$ versus $V_{p}$ at resonant point.

inset, we just plot Andreev coefficients at certain moments: $t_{0}=3 \pi / 4, t_{0}=0.95 \times 3 \pi / 4$ (a little smaller than $t_{0}$ ) and $t_{0}$ $=1.05 \times 3 \pi / 4$ (a little lager than $\left.t_{0}\right)$. We see that all three curves have double-peak feature (for the solid line the double-peak is barely seen). (3) The pumped current increases as the pumping amplitude $V_{p}$ increases (compare solid line, dotted line, and dashed line in Fig. 2). (4) For comparison, we also plot the pumped current $I^{\mathrm{N}}$ for N-SWNT-N system with $V_{p}=0.0014 \mathrm{~V}$ (long dashed line in Fig. 2). We see that it gives only one broad peak with small magnitude rather than two peaks. Due to the quantum interference between the direct reflection and the multiple Andreev reflection, the pumped current $I^{\mathrm{NS}}$ of N-SWNT-S is greatly enhanced and is much larger than the pumped current $I^{\mathrm{N}}$ of the same system when the superconducting lead becomes normal $^{18}$ (see the left inset of Fig. 2).

Now we consider a zigzag $(10,0)$ SWNT with $L=56$ layers of carbon atoms (total 560 atoms). Two pumping driving forces are added on the tube layers from 5 th to 8th layer and from 49th to 52nd layer. By adjusting $V_{0} \approx 2.10065 \mathrm{~V}$, one resonant level is available at $E_{F}=0$. In the calculation, we set $\phi=\pi / 2$ and $\Gamma_{R}=\Gamma_{L}=0.0136 \mathrm{eV}$. The results are plotted in Fig. 3. We see that the pumped current gives similar behavior to that of armchair SWNT system. The ratio of $I^{\mathrm{NS}} / I^{\mathrm{N}}$ is also about four in the weak pumping regime (see the inset of Fig. 3). Moreover there are several points worth mentioning. First, the pumped current can either be positive or negative even for the same SWNT but different energy levels or different phase differences (see Fig. 5 and Fig. 6). Comparing with armchair structure, the pumped current for zigzag structure reverses the direction. This is because the pumped current is not due to the external bias but cause by the pumping potentials. As a result, the pumped current is very sensitive to the system parameters. Second, the double-peak for the pumped current is asymmetric especially when the pumping amplitude is large (see the dashed line of Fig. 3). This is mainly due to the energy dependence of the self- 


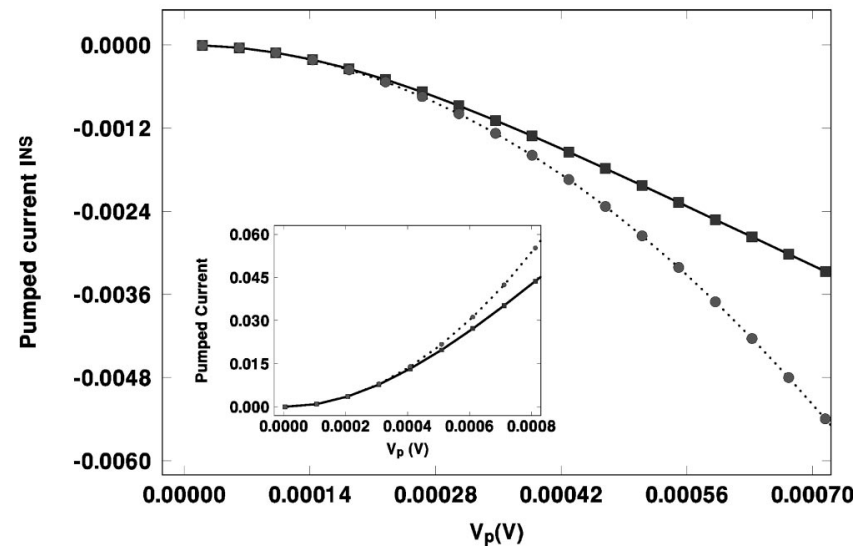

FIG. 4. The pumped current $I^{\mathrm{NS}}$ of the armchair SWNT as a function of pumping amplitude $V_{p}$ at $E_{F}=0$ (solid line). Other system parameters are the same as those in Fig. 2. Dotted line is plotted according to $I=-1.06 \times 10^{4} V_{p}^{2}$. Inset, the pumped current $I^{\mathrm{NS}}$ of the zigzag SWNT as a function of pumping amplitude $V_{p}$ at $E_{F}=0$ (solid line). System parameters are the same as those in Fig. 3. Dotted line is plotted according to $I=8.3758 \times 10^{5} V_{p}^{2}$.

energy. Actually, similar behavior has been found in Ref. 18 . However, the asymmetry is opposite, i.e., in Fig. 1 of Ref. 18, the left peak is higher than the right one while in our Fig. 3 the left peak is lower. Third, under the same system parameters, ${ }^{52}$ the pumped current of the zigzag structure is much larger (at least 10 times larger) than that of the armchair structure. This means that in order to obtain large pumped current, one should use zigzag nanotube instead of armchair nanotube. This may be useful for experimental study of the carbon nanotube pump.

Figure 4 gives the pumped current as a function of pumping amplitude $V_{p}$ at $E_{F}=0$ for armchair SWNT. Here the system parameters are the same as those in Fig. 2. We see that the pumped current increases quadratically at small pumping amplitude and then reaches linear regime for large pumping amplitude. In order to understand this figure, we also plot $I=-1.06 \times 10^{4} V_{p}^{2}$ in the same plot. We confirm that in the weak pumping regime the pumped current is proportional to the square of the pumping amplitude, but in the strong pumping regime the pumped current is linearly proportional to $V_{p}$ only. Similar conclusion can be drawn from the zigzag nanotube (see the inset of Fig. 4). Figure 5 and the inset of Fig. 5 present the pumped currents of armchair SWNT versus phase difference at different pumping amplitudes in the weak pumping regime and the strong pumping regime, respectively. We see that the pumped current is antisymmetric about $\phi=\pi$, just like the the result given by the experiments of Ref. 3 although superconducting lead was not used there. In the weak pumping regime, the sinusoidal behavior is clearly seen. In the strong pumping regime, however, we see strong deviation from the sinusoidal behavior (see inset of Fig. 5).

Now we examine another N-SWNT-S quantum pump using a zigzag $(10,0)$ tube with $L=92$ layers (total atoms 920). One gate is located from 10th to 28th layer and the other is located from 65 th to 83rd layer. By adjusting $V_{0}$ $\approx 2.5936 \mathrm{~V}$, we obtain two double degenerated resonant lev-

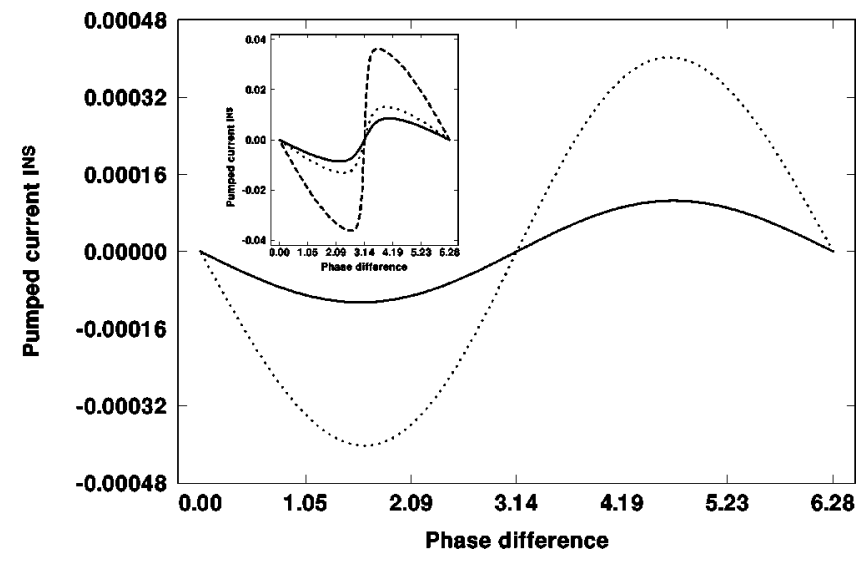

FIG. 5. The pumped current $I^{\mathrm{NS}}$ of the armchair SWNT as a function of phase difference $\phi$ at $E_{F}=0$. Main figure, the weak pumping regime with $V_{p}=0.0001 \mathrm{~V}$ (solid line) and $V_{p}$ $=0.0002 \mathrm{~V}$ (dotted line). Inset, the strong pumping regime with $V_{p}=0.0014 \mathrm{~V}$ (solid line), $V_{p}=0.002 \mathrm{~V}$ (dotted line), and $V_{p}$ $=0.005 \mathrm{~V}$ (dashed line). Other system parameters are the same as those in Fig. 2.

els at $E_{1}=-3.5495 \times 10^{-5} \mathrm{eV}$ and $E_{2}=3.5495 \times 10^{-5} \mathrm{eV}$. Hence, large Andreev reflections can occur near $E_{F}=E_{1}$ and $E_{F}=E_{2}$ with transmission coefficient equals to two (see the upper panel of Fig. 6). Here the Andreev reflection $T_{A}$ is due to different origin from that of Fig. 1. In Fig. 1, we have $E_{0}=0$ with $T_{A}=1$. If $E_{0}$ is nonzero the maximum Andreev reflection is less than one. In the strong tunneling regime which applies to our case, $\Gamma_{1}$ and $\Gamma_{2}$ are very small. To simplify the discussion, let us assume $\Gamma_{1}=\Gamma_{2} \ll 1$, then we have $^{47}$

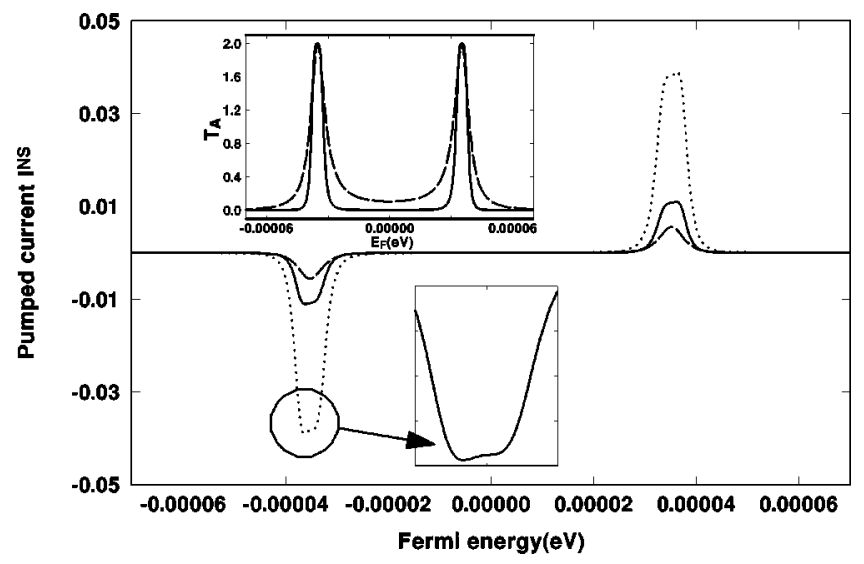

FIG. 6. The pumped current $I^{\mathrm{NS}}$ versus Fermi energy for the N-SWNT-S device at different pumping amplitudes: $V_{p}=1$ $\times 10^{-6} \mathrm{~V}$ (solid line), $V_{p}=2 \times 10^{-6} \mathrm{~V}$ (dotted line). The long dashed line is the pumped current $I^{\mathrm{N}}$ versus Fermi energy for the corresponding N-SWNT-N device at $V_{p}=1 \times 10^{-6} \mathrm{~V}$. The SWNT is a zigzag $(10,0)$ nanotube with length $L=92$ layers. The upper inset, Andreev reflection coefficient $T_{A}$ for N-SWNT-S device (solid line) and transmission coefficient $T$ for the corresponding N-SWNT-N device (long dashed line). The lower inset, the amplified figure of a pumped current peak. The other system parameters, $\phi=\pi / 2$ and $\Gamma_{R}=\Gamma_{L}=0.0136 \mathrm{eV}$. 


$$
T_{A}=\frac{\Gamma_{1}^{4}}{\left[4\left(E^{2}-E_{0}^{2}\right)^{2}+\Gamma_{1}^{4}+4 E_{0}^{2} \Gamma_{1}^{2}\right]} .
$$

At resonance, $T_{A}=\Gamma_{1}^{2} /\left[\Gamma_{1}^{2}+4 E_{0}^{2}\right]$. Hence when $E_{0}$ is larger than $\Gamma_{1}$, the Andreev reflection quickly goes to zero. However, if the chemical potential of the superconducting lead ( $\mu_{s}=0$ in our case) is right in the middle of two resonant levels $\left(E_{1}\right.$ and $\left.E_{2}\right)$, i.e., $\mu_{s}=\left(E_{1}+E_{2}\right) / 2$, then electron coming from normal lead with incident energy $E_{1}$ tunnels into the structure through the resonant level $E_{1}$ and Andreev reflected as a hole back to the quantum dot through the resonant level $E_{2}$ with a Copper pair created in the superconducting lead, giving rise to the complete transmission. This is why in the upper panel of Fig. 6 we have two transmission peaks with $T_{A}=2$ due to the double degeneracy. The pumped current as a function of Fermi energy is plotted in Fig. 6. For comparison, we also plot the transmission coefficient (long dashed line for normal structure and solid line for NS structure) in the upper panel of Fig. 6. The pumped current with $V_{p}=1 \times 10^{-6} \mathrm{~V}$ (long dashed line in Fig. 6) for the N-SWNT-N system with the same system parameters is also shown. Similar to Fig. 2 and Fig. 3, the pumped current also shows strong resonant behavior. It has large value near energies where the Andreev reflection peaks occur, while it diminishes quickly away from the peaks. The amplitude of pumped current also increases as the pumping amplitude $V_{p}$ increases. The striking feature here is that the pumped current peaks have opposite sign for the two energy levels similar to the case of the normal case. ${ }^{14}$ That means the pump has property that the DC current can flow out of the device from either electrodes by a slight change of electron energy. The physics behind this behavior of current reversal is similar to that of the normal case which is discussed in Ref. 14. For the normal case, the pumped current peaks is grouped in pairs with one positive peak and one negative peak. As the pumping amplitude increases, the pumped current peak height increases and the position is shifted away from the original static transmission peak. In another word, the two pumped current peaks repel each other. This repulsive behavior is understood using the phase diagram discussed in Ref. 14 by examining the position shifting of static transmission peaks upon varying the barrier height. For the normal CNT structure that was studied in Ref. 14, as one varies the barrier height, there is a global shifting for the position of the transmission peak which is directly responsible for the repulsive behavior (see Ref. 14 for details). For the NS CNT structure considered here, however, the two pumped current peaks stay in the same position as the pumped amplitude increases. This can still be described within the picture of phase diagram. ${ }^{14}$ We have confirmed that as the height of both barrier varies, the position of transmission peaks (upper panel of Fig. 6) does not change, i.e., global shift of transmission coefficient is forbidden since the middle point of two resonant levels is pinned at the superconducting condensate $\mu_{s}$ and due to the nature of Andreev reflection the transmission peaks must be symmetric about $\mu_{s}$. As a result, the repulsive behavior of pumped current peaks disappeared. The pumped current through two resonant levels has also

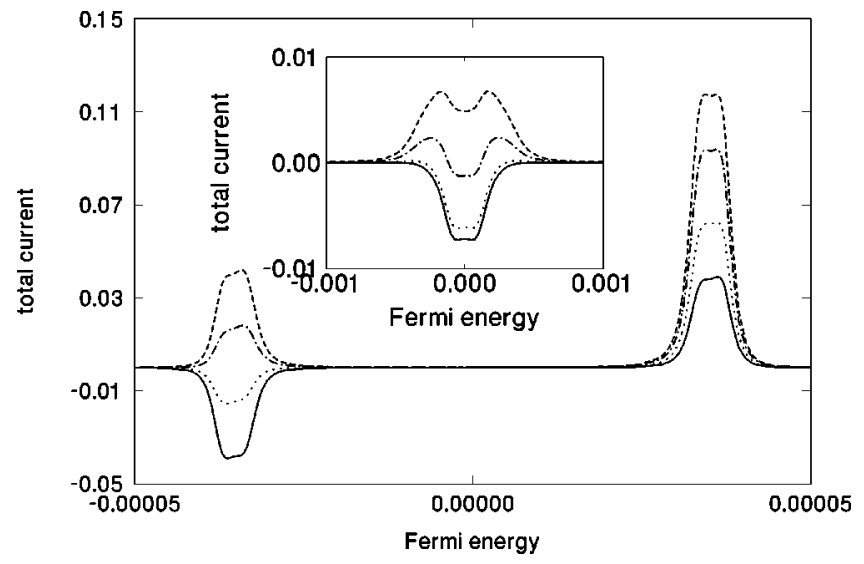

FIG. 7. The total current versus Fermi energy for the NS zigzag CNT device at at different external bias $V_{L}=0$ (solid line), $V_{L}$ $=0.3 \omega$ (dotted line), $V_{L}=0.7 \omega$ (dotted-dashed line), and $V_{L}=\omega$ (dashed line). Here $v_{p}=2 \times 10^{-6} \mathrm{~V}$ and the other parameters are the same as that of Fig. 6. Inset, the total current versus Fermi energy for the NS armchair CNT device at at different external bias $V_{L}=0$ (solid line), $V_{L}=0.01 \omega$ (dotted line), $V_{L}=0.1 \omega$ (dotteddashed line), and $V_{L}=\omega$ (dashed line). Here $v_{p}=0.0014 \mathrm{~V}$ and the other parameters are the same as that of Fig. 2.

been studied in Ref. 18 for one dimensional double barrier structure but only in the positive energy range. It remains to see whether one dimensional double barrier structure exhibits the current reversal behavior. We have also calculated the pumped current via two resonant levels for the one dimensional double barrier structure in the negative energy range as well which shows that the pumped current will not reverse the direction. Hence, the reversal of the pumped current direction is an unique feature of CNT as we have claimed and explained in Ref. 14. From the lower inset of Fig. 6, we see that the pumped current clearly consists of two asymmetric peaks. Finally, we notice that large pumped current is generated for very small pumping amplitude (compare Fig. 3 with Fig. 6). This is because in Fig. 6 the thickness of potential barrier and hence the effective potential barrier height is much higher than that in Fig. 3. As shown in Refs. 11 and 53 that the maximum pumped current can reach $1 / 2 \pi$ for extremely large barrier and in the strong pumping regime.

Now we examine the pumped current in the presence of external bias using Eq. (1). In the inset of Fig. 7, we plot the total current as a function of Fermi energy for armchair CNT at different external bias. We see that at small $V_{L}$, the total current is not very different from the pumped current at zero bias. As one increases the external voltage, we see some oscillation behavior that a positive double-peak shows up with a flat negative vally in the center. As one further increases the external bias the total current becomes positive with a broadened double-peak structure compared with that of the zero bias case. For the large external bias, the distance between the positions of double peaks approximately equal to the full width of the pumped current peak at zero bias. This can be qualitatively understood as follows. From Eq. (1) we see that for the positive external bias, $I_{v}$ is positive. This partially cancels the pumped current which is negative. Roughly speaking the current due to the external bias $I_{v}$ is 
proportional to the time averaged transmission coefficient at small voltage. For armchair CNT we consider here, the pumped current in the presence of external bias $I_{p}$ decays faster than $I_{v}$ which approximately has the shape of the transmission coefficient: a flat peak (see Fig. 1). As a result of the cancellation, $I_{v}$ decreases faster in the center of the peak region than that near the edge of the peak. Hence, from the inset of Fig. 7, we see that the position of the double peaks at large bias is near the position where $I_{p}$ at zero bias just decays to zero. Figure 7 depicts the total current as a function of Fermi energy for transmission through two energy levels for the same zigzag CNT shown in Fig. 6. The left peak of the total current roughly keeps the qualitative feature of the pumped current at small external bias. However, at large external bias, the total current becomes positive with an asymmetric peak opposite to that of the pumped current at zero bias. For the right peak of the total current, as one increases the external bias, the asymmetric feature of the pumped current at zero bias gradually diminishes. These behavior can also be understood qualitatively from the competition of between two terms in Eq. (1). For the zigzag CNT, the lineshape of $I_{v}$ is similar to that of $I_{p}$. As a result, we do not see the broadened double-peak structure shown in the inset of Fig. 7.

In summary, we have investigated the parametric pump of N-SWNT-S systems. By comparing with the parametric pump of N-SWNT-N system, we find that in the presence of

*Electronic address: jianwang@hkusub.hku.hk

${ }^{1}$ L. P. Kouwenhoven, A. T. Johnson, N. C. van der Vaart, C. J. P. M. Harmans, and C. T. Foxon, Phys. Rev. Lett. 67, 1626 (1991).

${ }^{2}$ H. Pothier, P. Lafarge, C. Urbina, D. Esteve, and M. H. Devoret, Europhys. Lett. 17, 249 (1992).

${ }^{3}$ M. Switkes, C. Marcus, K. Capman, and A. C. Gossard, Science 283, 1905 (1999).

${ }^{4}$ P. W. Brouwer, Phys. Rev. B 58, R10 135 (1998).

${ }^{5}$ I. L. Aleiner and A. V. Andreev, Phys. Rev. Lett. 81, 1286 (1998).

${ }^{6}$ F. Zhou, B. Spivak, and B. L. Altshuler, Phys. Rev. Lett. 82, 608 (1999).

${ }^{7}$ T. A. Shutenko, I. L. Aleiner, and B. L. Altshuler, Phys. Rev. B 61, 10366 (2000).

${ }^{8}$ Y. D. Wei, J. Wang, and H. Guo, Phys. Rev. B 62, 9947 (2000).

${ }^{9}$ I. L. Aleiner, B. L. Altshuler, and A. Kamenev, Phys. Rev. B 62, 10373 (2000).

${ }^{10}$ J. E. Avron, A. Elgart, G. M. Graf, and L. Sadun, Phys. Rev. B 62 , R10 618 (2000).

${ }^{11}$ Y. Levinson, O. Entin-Wohlman, and P. Wolfle, Physica A 302, 335 (2001).

${ }^{12}$ P. W. Brouwer, Phys. Rev. B 63, 121303 (2001); M. L. Polianski and P. W. Brouwer, ibid. 64, 075304 (2001).

${ }^{13}$ M. Moskalets and M. Buttiker, Phys. Rev. B 64, 201305 (2001).

${ }^{14}$ Y. D. Wei, J. Wang, H. Guo, and C. Roland, Phys. Rev. B 64, 115321 (2001).

${ }^{15}$ F. Renzoni and T. Brandes, Phys. Rev. B 64, 245301 (2001).

${ }^{16}$ Y. Makhlin and A. D. Mirlin, Phys. Rev. Lett. 87, 276803 (2001).

${ }^{17}$ C. S. Tang and C. S. Chu, Solid State Commun. 120, 353 (2001).

${ }^{18}$ J. Wang, Y. D. Wei, B. G. Wang, and H. Guo, Appl. Phys. Lett. 79, 3977 (2001). superconducting lead, the pumped currents is greatly enchanced due to the quantum interference of direct reflection and multiple Andreev reflection. In the weak pumping regime, the pumped current is proportional to the square of the pumping amplitudes but in the strong pumping regime, the dependence becomes linear. Hence large pumped current can be generated by increasing the pumping amplitude. When two level Andreev reflection occurs, the pumped current shows remarkable parity effect so that the pumped current at one resonant level has opposite direction of that of the other resonant level. In the presence of external bias, the current is driven by both the pumping potential and the external bias. In this case, we have also derived the formula for the NS system and applied the new formula to calculate the total current for CNT. Finally, our numerical results also show that the zigzag nanotube is a better candidate for the pumping device since it is more easily pumped than the armchair nanotube. In view of the S-SWNT-S structure studied in Ref. 36 , it is conceivable that the N-SWNT-S quantum molecular pump can be realized experimentally and the results presented here be verified.

\section{ACKNOWLEDGMENTS}

We gratefully acknowledge support by a RGC grant from the SAR Government of Hong Kong under Grant No. HKU 7091/01P.
${ }^{19}$ O. Entin-Wohlman, A. Aharony, and Y. Levinson, Phys. Rev. B 65, 195411 (2002).

${ }^{20}$ Q. F. Sun, J. Wang, and T. H. Lin, Phys. Rev. B 59, 3831 (1999).

${ }^{21}$ In the adiabatic approximation, the current due to the quasiparticle can be neglected when the Fermi energy is within the energy gap.

${ }^{22}$ O. Entin-Wolman and A. Aharony, Phys. Rev. B 66, 035329 (2002).

${ }^{23}$ J. E. Avron, A. Elgart, G. M. Graf, and L. Sadun, Phys. Rev. Lett. 87, 236601 (2001).

${ }^{24}$ M. Moskalets and M. Buttiker, Phys. Rev. B 66, 035306 (2002).

${ }^{25}$ M. L. Polianski, M. G. Vavilov, and P. W. Brouwer, Phys. Rev. B 65, 245314 (2002).

${ }^{26}$ B. G. Wang and J. Wang, Phys. Rev. B 66, 125310 (2002).

${ }^{27}$ M. G. Vavilov, V. Ambegaokar, and I. L. Aleiner, Phys. Rev. B 63, 195313 (2001).

${ }^{28}$ B. G. Wang, J. Wang, and H. Guo, Phys. Rev. B 65, 073306 (2002).

${ }^{29}$ M. Blaauboer, Phys. Rev. B 65, 235318 (2002).

${ }^{30}$ B. G. Wang and J. Wang, Phys. Rev. B 65, 233315 (2002).

${ }^{31}$ J. L. Wu, B. G. Wang, and J. Wang, cond-mat/0204570.

${ }^{32}$ Z. Yao, H. W. Ch. Postma, L. Balents, and C. Dekker, Nature (London) 402, 273 (1999).

${ }^{33}$ K. Tsukagoshi, B. W. Alphenaar and H. Ago (unpublished).

${ }^{34}$ S. J. Tans, M. H. Devoret, H. Dai, X. Thess, R. E. Smalley, L. J. Geerligs, and C. Dekker, Nature (London) 386, 174 (1997).

${ }^{35}$ S. Frank, P. Poncharal, Z. L. Wang, and W. A. de Heer (unpublished).

${ }^{36}$ A. F. Morpurgo, J. Kong, C. M. Marcus, and H. Dai, Science 286, 263 (2002). 
${ }^{37}$ D. H. Cobden, M. Bockrath, P. L. McEuen, A. G. Rinzler, and R. Z. Smalley, Phys. Rev. Lett. 81, 681 (1998).

${ }^{38}$ M. Mehrez, J. Taylor, H. Guo, J. Wang, and C. Roland, Phys. Rev. Lett. 84, 2682 (2000); H. Mehrez, H. Guo, J. Wang, and C. Roland, Phys. Rev. B 63, 245410 (2001).

${ }^{39}$ C. Roland, M. B. Nardelli, J. Wang, and H. Guo, Phys. Rev. Lett. 84, 2921 (2000); C. Roland, M. B. Nardelli, H. Guo, H. Mehrez, J. Taylor, J. Wang, and Y. D. Wei, Surf. Rev. Lett. 7, 637 (2000).

${ }^{40}$ D. Orlikowski, H. Mehrez, J. Taylor, H. Guo, J. Wang, and C. Roland, Phys. Rev. B 63, 155412 (2001).

${ }^{41}$ A. F. Andreev, Zh. Éksp. Teor. Fiz. 46, 1823 (1964) [Sov. Phys. JETP 19, 1228 (1964)].

${ }^{42}$ A. P. Jauho, N. S. Wingreen, and Y. Meir, Phys. Rev. B 50, 5528 (1994).

${ }^{43}$ Q. F. Sun, J. Wang, and T. H. Lin, Phys. Rev. B 58, 13007 (1998); 59, 13126 (1999).

${ }^{44}$ B. G. Wang, J. Wang, and H. Guo, Phys. Rev. Lett. 82, 398 (1999); J. Appl. Phys. 86, 5094 (1999).

${ }^{45}$ We have omitted the factor of 2 for spin.

${ }^{46}$ T. Gramespacher and M. Buttiker, Phys. Rev. B 61, 8125 (2000).
${ }^{47}$ J. Wang, Y. D. Wei, H. Guo, Q. F. Sun, and T. H. Lin, Phys. Rev. B 64, 104508 (2001).

${ }^{48}$ Y. Nambu, Phys. Rev. 117, 648 (1960).

${ }^{49}$ X. Blase, L. X. Benedict, E. L. Shirley, and S. G. Louie, Phys. Rev. Lett. 72, 1878 (1994); Y. A. Krotov, D. H. Lee, and S. G. Louie, ibid. 78, 4245 (1997); L. Chico, V. H. Crespi, L. X. Benedict, S. G. Louie, and M. L. Cohen, ibid. 76, 971 (1996); V. H. Crespi, M. L. Cohen, and A. Rubio, ibid. 79, 2093 (1997); L. Chico, M. P. Lopez Sancho, and M. C. Munoz, ibid. 81, 1278 (1996); L. Chico, L. X. Benedict, S. G. Louie, and M. L. Cohen, Phys. Rev. B 54, 2600 (1996).

${ }^{50}$ M. BuongiornoNardelli, Phys. Rev. B 60, 7828 (1999).

${ }^{51}$ Y. D. Wei, J. Wang, H. Guo, H. Mehrez, and C. Roland, Phys. Rev. B 63, 195412 (2001).

${ }^{52}$ In fact, the barrier thickness and barrier height of armchair nanotube are larger than that of zigzag nanotube which favor the pumping of armchair nanotube. Yet the pumped current for zigzag nanotube is much larger than that of armchair nanotube with the same pumping amplitude.

${ }^{53}$ J. Wang and B. G. Wang, Phys. Rev. B 65, 153311 (2002). 